\title{
Temperature-dependent Spectral Emission of Hexagonal Boron Nitride Quantum Emitters on Conductive and Dielectric Substrates
}

\author{
Hamidreza Akbari $\odot,{ }^{1}$ Wei-Hsiang Lin, ${ }^{1}$ Benjamin Vest, ${ }^{1,2}$ Pankaj K. Jha, ${ }^{1}$ and Harry A. Atwater ${ }^{1, *}$ \\ ${ }^{1}$ Thomas J. Watson Laboratories of Applied Physics, California Institute of Technology, Pasadena, California, \\ 91125, USA \\ ${ }^{2}$ Institut d'Optique Graduate School, CNRS, Laboratoire Charles Fabry, Université Paris-Saclay, 91127, \\ Palaiseau, France
}

(Received 12 October 2020; revised 4 December 2020; accepted 9 December 2020; published 20 January 2021)

\begin{abstract}
We report a reduction in the linewidth and suppression of spectral diffusion of quantum emitters in hexagonal boron nitride supported on a conductive substrate. We observe a temperature-dependent reduction in the spectral emission linewidth for CVD-grown and exfoliated crystals on conductive ITO relative to those seen on silicon dioxide $\left(\mathrm{SiO}_{2}\right)$ substrates. We show that the inhomogeneous linewidth can be suppressed by $45 \%$ as a result of using a conductive substrate. We investigate the zero-phonon line profile at temperatures ranging from 4 to $300 \mathrm{~K}$ and decompose the effects of thermal broadening and spectral diffusion at each temperature by Voigt fitting. The temperature dependence of homogeneous and inhomogeneous components of the broadening is discussed.
\end{abstract}

DOI: 10.1103/PhysRevApplied.15.014036

\section{INTRODUCTION}

Single-photon emitters are essential building blocks of future quantum-information technologies [1]. Many different single-photon sources have been proposed, including solid-state lattice hosts such as epitaxially grown quantum dots [2] and atomic defects in diamond [3]. Each emitter type is characterized by spectral diffusion, i.e., temporal variations in emission energy around a nominal value, which poses a challenge to the use of solid-state quantum emitters as sources of indistinguishable single photons [4,5]. Single-photon emitters in two-dimensional van der Waals materials have garnered considerable attention due to their promising photophysical properties and the possibility for on-chip integration [6,7]. Among them, color centers in two-dimensional dielectric hexagonal boron nitride ( $h$-BN) promise a robust medium for the generation of quantum light with potential for room-temperature operation [8-10]. These color centers exhibit a Debye-Waller factor [11] $>80 \%$, a brightness on par with the brightest sources of single photons $[6,9,12]$, and linearly polarized emission [11,13], all at room temperature. These unique properties have the potential for quantum-communication applications such as quantum-key distribution in which a bright source of linearly polarized single photons is required $[14,15]$.

Despite these inherent benefits, several challenges facing $h$-BN color centers need to be resolved to make them suitable for quantum information and sensing applications.

\footnotetext{
*haa@caltech.edu
}

The atomic structure of the defects underlying the color centers is under debate [16-21]. As a result, the electronic and spin $[22,23]$ characteristics of these emitters are still not clear, and methods to deterministically create emitters in $h$-BN crystals are not well developed [24-26]. Furthermore, instabilities, such as blinking [12,27,28], bleaching [29], and spectral diffusion, [22,29-31] can yield interesting physical insights but ultimately represent significant practical drawbacks for the use of $h$-BN color centers as a source of indistinguishable single photons. Recently, Fourier-transform-limited spectral linewidths have been reported for $h$-BN emitters at both 4 and $300 \mathrm{~K}[32,33]$. This observation of Fourier-transform-limited linewidth has a spectral diffusion-limited time window of $30 \mathrm{~ms}$, inhibiting indistinguishability of the spectral line in consecutive measurements.

Spectral diffusion is the temporal change in energy of emitted photons due to a change in the local electric field distribution of the emitter. This temporal change in energy manifests itself as a series of jumps of the zero phonon line (ZPL) in measurements with time resolution high enough to capture microsecond-to-millisecond scale changes in the energy. In lower time-resolution measurements, where the acquisition time is longer than the time between spectral jumps, it can be observed as a broadening of the spectral lines [5,34]. The change in the local electric field is thought to be due to either trapping and detrapping of charge close to the position of the active photon emitter or due to charges hopping from one site to another in the host lattice. The timescale of these spectral jumps in $h$-BN is on the order of microseconds to milliseconds 
$[33,34]$. Thermally grown silicon dioxide $\left(\mathrm{SiO}_{2}\right)$ on silicon, the material widely used as a substrate for graphene and other two-dimensional materials $[35,36]$ due to its low surface roughness, is an insulator prone to charge traps, a source of spectral diffusion for color-center emitters. It was reported that fluctuating charge environment can affect spectral diffusion, photon statistics purity, and decay lifetime of excitonic single-photon emitters such as emitters in monolayer $\mathrm{WSe}_{2}$ and CdSe quantum dots [37-39]. Although another study noted the effect of substrate choice on emission in $h$-BN [22], the role of the substrate in determining the spectral linewidth has not been addressed.

In this paper, we report reduced linewidth and spectral diffusion when conductive transparent material ITO is used as a substrate for $h$-BN color centers. As shown in Fig. 1, a transparent conductive material such as ITO lacks charge traps on its surface and can provide a conductive pathway for charges trapped in $h$-BN to be removed. These removed charges can then screen trapped and mobile charges from the $h$-BN color centers, thereby suppressing

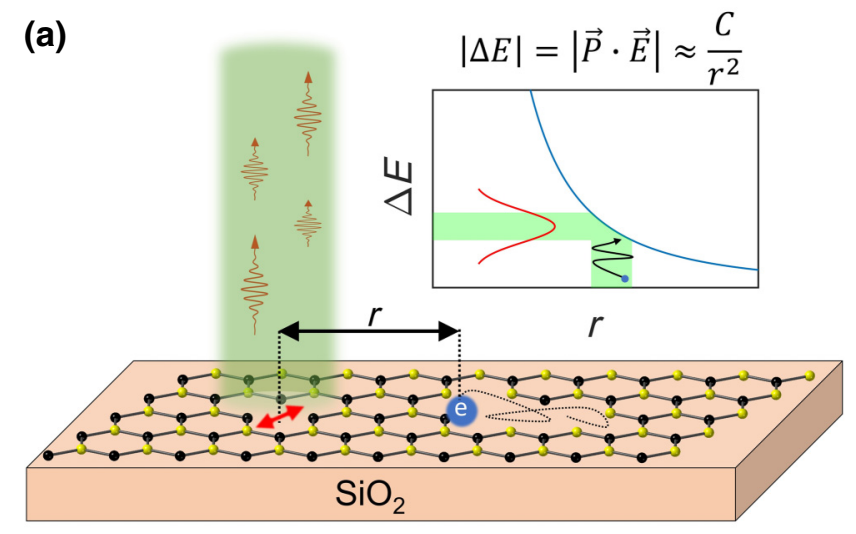

(b)

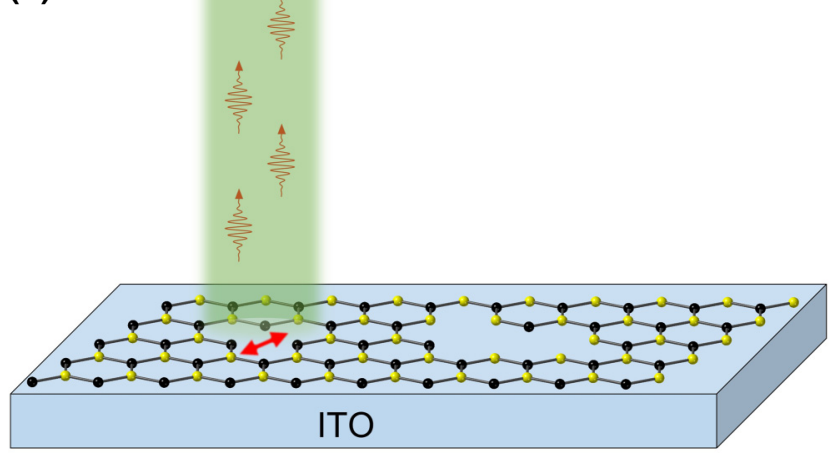

FIG. 1. Schematic of substrate effect on spectral diffusion of single-photon emitters in $h$-BN. (a) $h$-BN emitter on an insulating $\mathrm{SiO}_{2}$ substrate is prone to larger spectral diffusion due to charge traps in $h$-BN, $\mathrm{SiO}_{2}$, and their interface. The inset shows Starkshift dependence on distance of nearby charges and how changes in the local charge distribution can broaden the linewidth. (b) $h$ BN emitter on an ITO substrate shows narrower linewidth as a result of fewer charge traps in $h$-BN, ITO, and their interface. spectral diffusion. Unlike many other conventional metals, ITO is transparent in the visible wavelength range and has a positive real part of its dielectric permittivity. As a consequence, it does not quench luminescence emission in proximity to an emitter. Spectral diffusion represents an obstacle to the achievement of stable sources of indistinguishable photons. Thus, developing methods to suppress spectral diffusion and increase the coherence time [34] for $h$-BN single-photon emitters is a helpful step towards the use of color centers as sources of indistinguishable photons in quantum applications based on single or entangled photons [14,40].

\section{RESULTS AND DISCUSSION}

\section{A. Temperature dependence of ZPL in bulk $h$-BN}

We start our analysis by investigating color centers in bulk $h$-BN. These centers reflect the intrinsic properties of $h$-BN emitters as they are inside the $h$-BN crystal and isolated from the environment. To fabricate bulk $h$-BN samples, we mechanically cleaved a thick flake from an $h$-BN crystal. AFM measurements show a thickness of $839 \mathrm{~nm}$ (see Fig. S1 within the Supplemental Material [41]). Emitters are initially characterized in a homebuilt confocal microscope at room temperature and then transferred to a variable temperature microscope employing a closed-cycle helium cryostat to perform temperaturedependent emission measurements from 4 to $300 \mathrm{~K}$. (See the Supplemental Material [41] for a complete description of the sample preparation method and experimental setup.) We use a 532-nm continuous-wave laser to excite the emitters and the PL is collected by a microscope objective through a tunable bandpass filter and coupled to either a spectrometer or a Hanbury-Brown-Twiss interferometer to perform an intensity autocorrelation measurement. To locate and observe emitters in bulk $h$-BN, a PL map of the sample is acquired (see Fig. 2). The spectral and spatial features of 12 selected emitters are identified. For the rest of the discussion, we focus on two representative emitters from this group.

PL spectroscopy and intensity correlation measurements $\left[g^{(2)}\right]$ of these two emitters are shown in Fig. 2. No background subtraction is performed on $g^{(2)}$ data and the measurements are fitted by a double exponential model function:

$$
g^{2}(\tau)=1-b\left[(1+a) e^{-\frac{|\tau|}{T_{1}}}-a e^{-\frac{|\tau|}{T_{2}}}\right] .
$$

Both photon-flux statistics show a $g^{2}(0)$ dip with values of 0.20 and 0.28 , confirming that both emitters are single-photon emitters. They exhibit similar decay lifetimes $\left(T_{1} \approx 1.3 \mathrm{~ns}\right)$ but their bunching time scales $\left(T_{2}\right)$ are different (see Fig. S5 within the Supplemental Material [41]). A similar decay lifetime suggests that these emitters, 

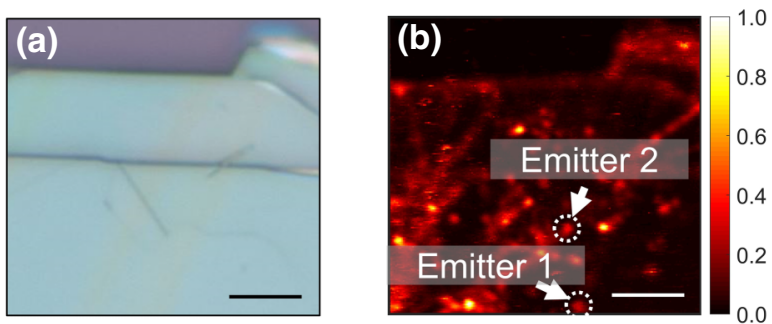

(c)

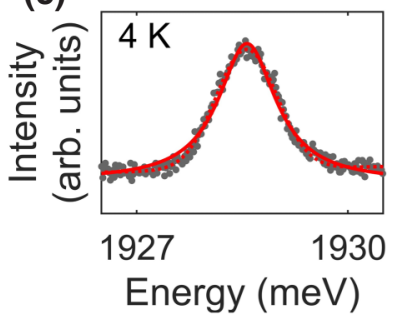

(d)

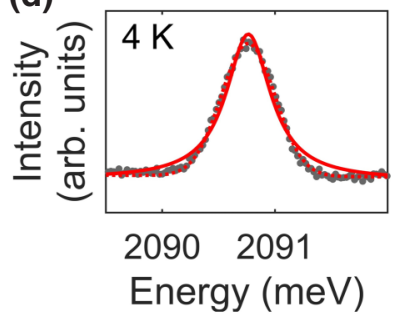

(e)

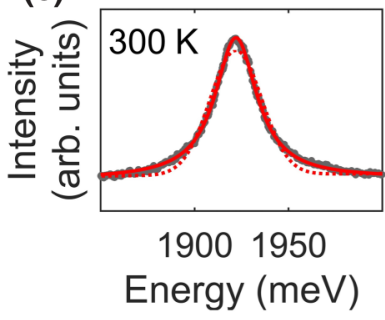

(g)

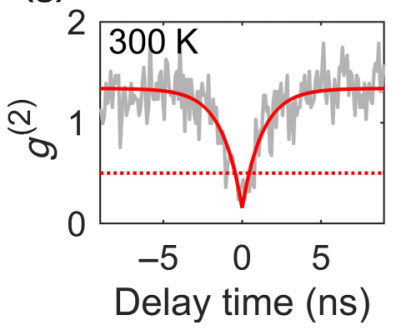

(f)

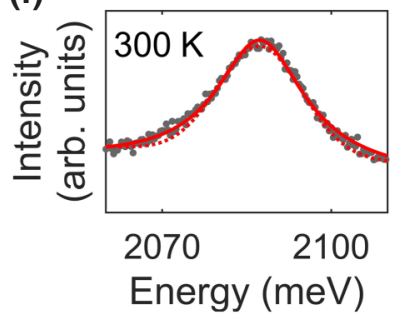

(h)

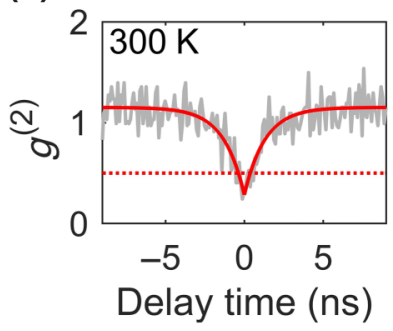

FIG. 2. Quantum emitters in bulk $h$-BN. (a) Microscope image and (b) PL map of a bulk $h$-BN crystal. The arrows show the position of two emitters under study; scale bar corresponds to $5 \mu \mathrm{m}$. (c) $4 \mathrm{~K}$ and (e) $300 \mathrm{~K}$ spectra of ZPL of emitter 1 . Solid line shows Lorentzian and the dashed line shows Gaussian fits (g) shows photon antibunching for emitter 1. (d), (f), and (h) show the same plots for emitter 2 .

in principle, should exhibit similar Fourier-transformlimited linewidth if no extrinsic broadening mechanism is operative. The difference in the bunching shoulder can be explained by the difference in the blinking characteristics of these emitters [42]. We notice that despite a nearly twofold difference in the linewidth between these emitters both at room temperature and $4 \mathrm{~K}$, the lineshapes of both emitters are similar throughout; namely, both emitters exhibit a Lorentzian lineshape at $300 \mathrm{~K}$ and a Gaussian lineshape at $4 \mathrm{~K}$ as shown in Fig. 2.

To further investigate the effect of temperature, the ZPL is studied at $4,10,20,40,80,160$, and $300 \mathrm{~K}$ and the

results are illustrated in Fig. 3. The evolution of the spectral line position and shape as a function of temperature is shown in Figs. 3(a) and 3(b) for the two emitters. The linewidths for both emitters remain largely independent of temperature up to the transition temperature $\left(T_{\text {tr }}\right)$ at $40 \mathrm{~K}$ and increase significantly at temperatures above $T_{\text {tr }}$ [see Fig. 3(c)]. These observations also confirm two different modes of broadening for emitters in bulk $h$-BN: a temperature-independent broadening mechanism at $T<$ $T_{\text {tr }}$ and a temperature-dependent broadening at $T>T_{\text {tr }}$. Similar behavior has been observed in other quantum emitters and is attributed to spectral diffusion and thermal phonon interactions, respectively [5,34]. As an inhomogeneous broadening mechanism, spectral diffusion results in Gaussian lineshapes [5]. In contrast, thermal phonon broadening is a result of quadratic interaction between thermal acoustic phonons and the emitter and will result in a homogeneous broadening and a Lorentzian line profile $[5,43]$. As the temperature increases, the line profile also becomes less Gaussian and more Lorentzian. To analyze the line profile, we fit a convolution of Gaussian and Lorentzian lineshapes to the line profile, also known as a Voigt profile:

$$
\begin{gathered}
I_{V}(\omega)=\frac{A \operatorname{Re}[w(z)]}{\sigma \sqrt{2 \pi}} \\
w(z)=e^{-z^{2}} \operatorname{erfc}(-i z) z=\frac{\omega-\omega_{0}+i \gamma}{\sigma \sqrt{2}},
\end{gathered}
$$

where $A$ is amplitude and $\mathrm{FWHM}_{\text {Gaussian }}=2 \sigma \sqrt{2 \ln (2)}$ and FWHM $_{\text {Lorentzian }}=2 \gamma$ [34].

By fitting a Voigt profile to the measured spectral lines, we can measure the FWHM of each Gaussian and Lorentzian component of the line profile at each temperature and deduce the homogeneous and inhomogeneous FWHM. As shown in the inset of Fig. 3(e) the inhomogeneous broadening is independent of temperature up to $T_{\text {tr }}$ for both emitters. At temperatures above $T_{\text {tr }}$, the Voigt fit does not show any significant Gaussian component and is predominantly Lorentzian. Therefore, for all temperatures above $T_{\text {tr }}$, we set the value of the Gaussian FWHM to the measured average value of the inhomogeneous linewidth below $T_{\text {tr }}$, and use a Voigt profile fit to find the Lorentzian FWHM component $[5,34]$. At $T_{\text {tr }}=40 \mathrm{~K}$, the Gaussian (inhomogeneous) and Lorentzian (homogeneous) line profiles are almost equal for each emitter. We can also observe two separate peaks for emitter 1 . We attribute the weaker peak to spectral jumps at timescales shorter than the 1-min exposure time of our spectral measurement, as the intensity of the second peak relative to the first peak varies significantly in time for time scales above $1 \mathrm{~min}$ (see Fig. S6 within the Supplemental Material [41]). 

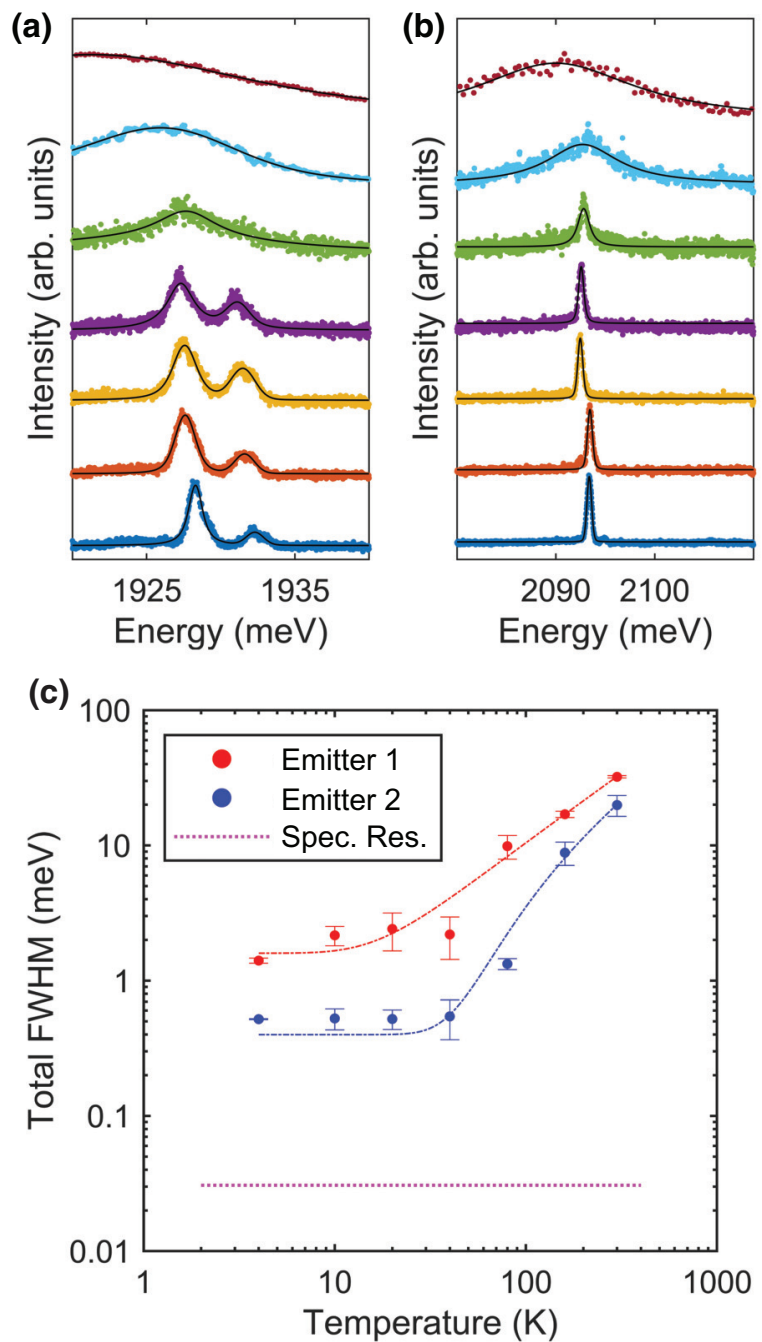

(d)

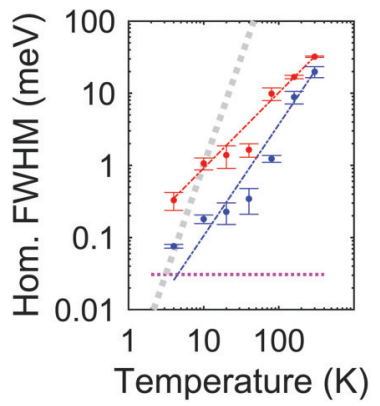

(e)

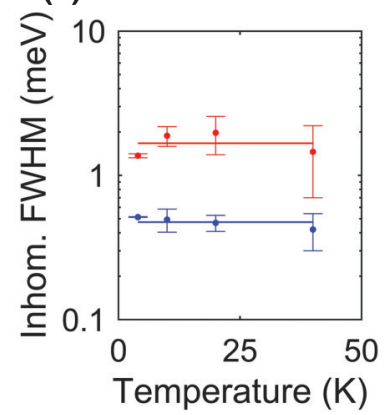

FIG. 3. Temperature dependence of ZPL for emitters in bulk $h$-BN. Effect of temperature on lineshape for emitter 1 (a) and emitter 2 (b) from 4 to $300 \mathrm{~K}$. (c) Linewidth against temperature for the two emitters under study. Linear fits for temperatures below and above $40 \mathrm{~K}$ are shown; dashed lines are fits with a model Eq. (4), and the dashed magenta line shows the spectral resolution of the spectrometer used in this study. In (d) and (e) homogeneous and inhomogeneous components of linewidth extracted by Voigt fit are plotted as a function of temperature. The slope of power-law fit for homogeneous linewidth for emitter 1 is $1.05 \pm 0.21$ and for emitter 2 is $1.54 \pm 0.38$. The dotted gray line in (d) corresponds to $T^{3}$ dependence of linewidth on temperature.
In Fig. 3(d) the homogeneous linewidth is plotted as a function of temperature in a log-log plot. The results for both emitters can be fitted by a line that corresponds to a power-law dependence of linewidth on the temperature, where the power is the slope of the fit line. We observe here a nearly linear dependence of the linewidth on temperature for both emitter 1 and emitter 2. This observation contrasts with other studies for $h$-BN and diamond quantum emitters, which observe a power-law dependence of the third power form $[5,34,44,45]$. We speculate that this difference in observed power-law temperature dependence may be the result of different structural and electronic configurations of the emitter color centers, a consequence of the diversity of defects seen in $h$-BN $[33,46]$. A similar linear dependence of linewidth on temperature is observed for excitons in two-dimensional transition metal dichalcogenides [47-49]. Using a similar approach to model $h$-BN, the total linewidth $\gamma_{\text {total }}$ of the ZPL can be written as the sum of an inhomogeneous and homogeneous contributions:

$$
\begin{aligned}
\gamma_{\text {total }}= & \gamma_{\text {inhom. }}+\frac{b}{\exp (\theta / k T)-1} \\
& \approx \gamma_{\text {inhom. }}+\frac{k b}{\theta} T,
\end{aligned}
$$

where " $\theta$ " is the acoustic phonon energy and " $b$ " is a constant factor. In a regime where the phonon energy is small compared to $k T$, a linear dependence of linewidth on temperature can be expected. A recent study [50] on exciton-phonon interaction in $h$-BN shows a similar linear trend in the exciton-phonon scattering rate in $h$-BN.

We also investigate the low-frequency spectral diffusion characteristics of these two emitters (see Fig. S6 within the Supplemental Material [41]). Emitter 1, which has a broader linewidth, exhibits larger spectral instability. Emitter 2, which has a narrower linewidth, exhibits fewer spectral jumps per unit of time, as can be seen from the Fourier transform of the time-evolution trace of the peak position of the emitter's ZPL. We confirm this result for the other ten emitters (see Fig. S7 within the Supplemental Material [41]) and reveal a correlation between the inhomogeneous linewidth and the average of the Fourier transform of the measured spectral diffusion. This observation also suggests that the origin of inhomogeneous broadening in our measurements is most likely spectral diffusion.

Since inhomogeneous broadening is the limiting factor to reach Fourier-transform-limited linewidths at cryogenic temperature in $h$-BN emitters, we also perform a statistical analysis of the inhomogeneous broadening [see Figs. 4(a) and 4(b)]. For the bulk $h$-BN sample, we see that the minimum inhomogeneous FWHM is $0.21 \mathrm{meV}$ (while the spectral resolution limit for our apparatus is $0.03 \mathrm{meV}$ ) and the median value is $0.41 \mathrm{meV}$. 


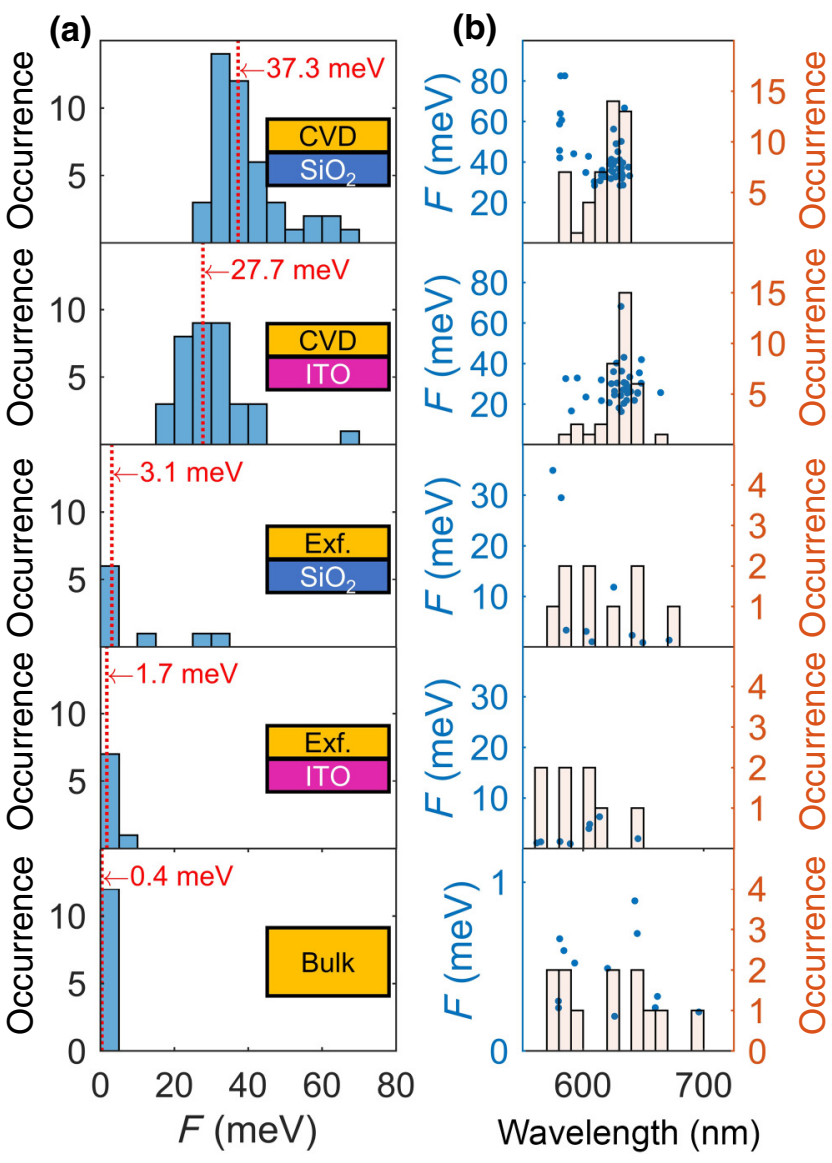

FIG. 4. Effect of $h$-BN crystal and substrate type on inhomogeneous broadening. Panel (a) is a histogram of linewidths for emitters and (b) is the scatter plot of linewidth against the ZPL wavelength and histogram of the number of occurrences of a ZPL wavelength, from top to bottom this figure shows the result for CVD film on $\mathrm{SiO}_{2}$ substrate, CVD film on ITO substrate, exfoliated flake on $\mathrm{SiO}_{2}$ substrate, exfoliated flake on ITO substrate, and bulk $h$-BN crystal, respectively. The red dashed line shows the median of inhomogeneous linewidth for each sample.

\section{B. Low-temperature broadening and the substrate effects}

Bulk $h$-BN is shown to have stable emitters with relatively narrow linewidths. However, to explore the full potential of the two-dimensional nature of $h$-BN, and to form layered heterostructures, emitters in samples with thicknesses of a few layers are of considerable interest. A drawback of emitters hosted in a few-layer $h$-BN samples is susceptibility to the environment. This environmental effect can manifest itself as increased spectral diffusion and a decreased coherence time. Therefore, our investigation focuses on $h$-BN layers less than $10 \mathrm{~nm}$ thick. CVD is a widely employed method to grow thin films of $h$-BN and provides the ability to grow $h$-BN with an arbitrary number of layers on a scale of millimeters to centimeters.
Another method for synthesis of few-layer $h$-BN crystal is by mechanical exfoliation, but this method does not provide large films of $h$-BN and lacks precise control over the thickness of the exfoliated flakes and trial and error is used to achieve the desired thickness. Nonetheless, this method can result in two-dimensional flakes with larger grain sizes.

To test the effect of measurement environment and $h$ BN crystal morphology on inhomogeneous broadening we transfer 5-nm-thick CVD-grown $h$-BN films from the copper growth substrate onto $\mathrm{SiO}_{2}$ and ITO substrates using wet-transfer techniques (for details about the growth method, see Ref. [51] and the Supplemental Material [41]). We also exfoliate a 5-nm-thick $h$-BN onto $\mathrm{SiO}_{2}$ substrate and an 8 -nm-thick $h$-BN onto the ITO substrate. We observe that for CVD-grown $h$-BN, linewidths at $4 \mathrm{~K}$ are almost 1 order of magnitude larger than those observed in the exfoliated samples with the same thickness and 2 orders of magnitude larger than emitters in bulk $h$-BN (see Fig. 4). We attribute this difference in linewidth to smaller grain sizes in the CVD films, which are expected to have a larger charge-trap density than $h$-BN bulk crystals. Comparing different substrates, for CVD $h$-BN-atop-ITO samples, we observe a $27 \%$ reduction of the inhomogeneous FWHM compared to CVD $h$-BN-atop- $\mathrm{SiO}_{2}$, (from 37.3 to $27.7 \mathrm{meV}$ ). For exfoliated $h$-BN-atop-ITO samples, we observe a $45 \%$ reduction of inhomogeneous FWHM compared to exfoliated $h$-BN-atop-SiO ${ }_{2}$, from 3.1 to $1.7 \mathrm{meV}$ ). This observation confirms our hypothesis that using a conductive substrate suppresses spectral diffusion,

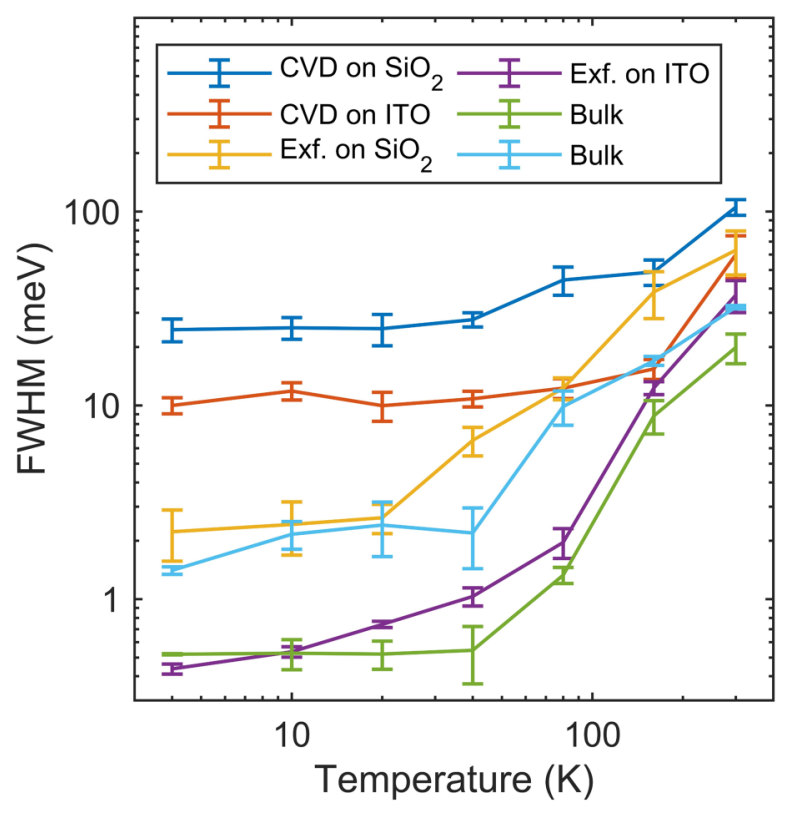

FIG. 5. Temperature dependence of ZPL FWHM for various emitters in bulk $h$-BN, 5-nm-thick exfoliated $h$-BN on $\mathrm{SiO}_{2}, 8$ nm-thick exfoliated $h$-BN on ITO, 5-nm-thick CVD $h$-BN on $\mathrm{SiO}_{2}$, and 5-nm-thick CVD $h$-BN on ITO. The result illustrates the total FWHM for temperatures from 4 to $300 \mathrm{~K}$. 
which is attributed to conductive pathways for transport of charge away from the vicinity of the emitter, as depicted in Fig. 1. Furthermore, no correlation between inhomogeneous broadening and the position of the ZPL wavelength is observed (see Fig. 4, right panel). This indicates that the inhomogeneous broadening mechanism is independent of defect structure and emission wavelength for exfoliated, CVD, and bulk $h$-BN.

To better understand the effect of ambient temperature, we measure the emitter temperature-dependent linewidth for CVD and few-layer exfoliated $h-\mathrm{BN}$ on $\mathrm{SiO}_{2}$ and ITO substrates (see Fig. 5 and Fig. S9 within the Supplemental Material [41]). Unlike bulk $h$-BN, the transition from spectral diffusion broadened to thermally broadened linewidth in CVD $h$-BN does not happen at $T_{\mathrm{tr}}=40 \mathrm{~K}$ but at around $T_{\text {tr }}=160 \mathrm{~K}$ (see solid red and dark blue lines). This is a direct consequence of the larger spectral diffusion observed in CVD $h$-BN. For few-layer exfoliated $h$-BN, we observe a larger change in the FWHM with temperature, and a transition temperature below $40 \mathrm{~K}$, despite a larger inhomogeneous linewidth (yellow and purple curves). We suggest that this may be due to the proximity of emitters to the $h$-BN flake surface, and acoustic coupling to the substrate.

\section{CONCLUSIONS}

In summary, we study the photodynamics of $h$-BN single-photon emitters in a diverse array of sample environments. We find both a temperature-dependent and temperature-independent regime for emitter linewidth that we attribute to thermally generated phonon interactions and spectral diffusion, respectively, as two mechanisms responsible for ZPL emission broadening. We suggest that among these two regimes, the spectral diffusion-limited linewidth is dominant at cryogenic temperatures and is independent of temperature, while thermal phonon interaction is dominant at temperatures close to room temperature and increases with a power-law dependence on temperature, with power close to unity. It is worthwhile to note that we find that the sample morphology and thickness, in the various configurations we investigate, affects the inhomogeneous linewidth, suggesting that the linewidth depends on both the local structure of $h$-BN and the surrounding environment in the crystal. In particular, we show that increasing the carrier density in the local environment of the emitter, achieved using a conductive substrate, significantly reduces the inhomogeneous linewidth. This is of interest for all applications that aim for spectral stability of color-center-based single-photon sources.

\section{ACKNOWLEDGMENTS}

This work is supported by the "Photonics at Thermodynamic Limits" Energy Frontier Research Center funded by the U.S. Department of Energy, Office of Science,
Office of Basic Energy Sciences under Award Number DESC0019140. We thank Phillip Jahelka for the discussion and Komron Shayegan and Arun Nagpal for feedback on the paper.

[1] M. D. Eisaman, J. Fan, A. Migdall, and S. V. Polyakov, Single-photon sources and detectors, Rev. Sci. Instrum. 82, 071101 (2011).

[2] Y. Arakawa and M. J. Holmes, Progress in quantum-dot single photon sources for quantum information technologies: a broad spectrum overview, Appl. Phys. Rev. 7, 021309 (2020).

[3] I. Aharonovich, S. Castelletto, D. A. Simpson, C. H. Su, A. D. Greentree, and S. Prawer, Diamond-Based single-photon emitters, Reports Prog. Phys. 74, 076501 (2011).

[4] M. Holmes, S. Kako, K. Choi, M. Arita, and Y. Arakawa, Spectral diffusion and Its influence on the emission linewidths of site-controlled $\mathrm{GaN}$ nanowire quantum dots, Phys. Rev. B 92, 115447 (2015).

[5] E. Neu, C. Hepp, M. Hauschild, S. Gsell, M. Fischer, H. Sternschulte, D. Steinmüller-Nethl, M. Schreck, and C. Becher, Low-Temperature investigations of single silicon vacancy colour centres in diamond, New J. Phys. 15, 043005 (2013).

[6] I. Aharonovich, D. Englund, and M. Toth, Solid-State single-photon emitters, Nat. Photonics 10, 631 (2016).

[7] I. Aharonovich and M. Toth, Quantum emitters in Two dimensions, Science (80-.) 358, 170 (2017).

[8] T. T. Tran, C. Elbadawi, D. Totonjian, C. J. Lobo, G. Grosso, H. Moon, D. R. Englund, M. J. Ford, I. Aharonovich, and M. Toth, Robust multicolor single photon emission from point defects in hexagonal boron nitride, ACS Nano 10, 7331 (2016).

[9] M. Kianinia, B. Regan, S. A. Tawfik, T. T. Tran, M. J. Ford, I. Aharonovich, and M. Toth, Robust solid-state quantum system operating at $800 \mathrm{~K}$, ACS Photonics 4, 768 (2017).

[10] J. D. Caldwell, I. Aharonovich, G. Cassabois, J. H. Edgar, B. Gil, and D. N. Basov, Photonics with hexagonal boron nitride, Nat. Rev. Mater. 4, 552 (2019).

[11] T. T. Tran, K. Bray, M. J. Ford, M. Toth, and I. Aharonovich, Quantum emission from hexagonal boron nitride monolayers, Nat. Nanotechnol. 11, 37 (2016).

[12] L. J. Martínez, T. Pelini, V. Waselowski, J. R. Maze, B. Gil, G. Cassabois, and V. Jacques, Efficient single photon emission from a high-purity hexagonal boron nitride crystal, Phys. Rev. B 94, 121405(R) (2016).

[13] A. L. Exarhos, D. A. Hopper, R. R. Grote, A. Alkauskas, and L. C. Bassett, Optical signatures of quantum emitters in suspended hexagonal boron nitride, ACS Nano 11, 3328 (2017).

[14] J. L. O’Brien, A. Furusawa, and J. Vučković, Photonic quantum technologies, Nat. Photonics 3, 687 (2009).

[15] B. Lounis and M. Orrit, Single-Photon sources, Reports Prog. Phys. 68, 1129 (2005).

[16] S. A. Tawfik, S. Ali, M. Fronzi, M. Kianinia, T. T. Tran, C. Stampfl, I. Aharonovich, M. Toth, and M. J. Ford, FirstPrinciples investigation of quantum emission from $\mathrm{HBN}$ defects, Nanoscale 9, 13575 (2017). 
[17] N. Chejanovsky, M. Rezai, F. Paolucci, Y. Kim, T. Rendler, W. Rouabeh, F. Fávaro De Oliveira, P. Herlinger, A. Denisenko, S. Yang, I. Gerhardt, A. Finkler, J. H. Smet, and J. Wrachtrup, Structural attributes and photodynamics of visible spectrum quantum emitters in hexagonal boron nitride, Nano Lett. 16, 7037 (2016).

[18] G. Grosso, H. Moon, B. Lienhard, S. Ali, D. K. Efetov, M. M. Furchi, P. Jarillo-Herrero, M. J. Ford, I. Aharonovich, and D. Englund, Tunable and high-purity room temperature single-photon emission from atomic defects in hexagonal boron nitride, Nat. Commun. 8, 705 (2017).

[19] F. Hayee, L. Yu, J. L. Zhang, C. J. Ciccarino, M. Nguyen, A. F. Marshall, I. Aharonovich, J. Vučković, P. Narang, T. F. Heinz, and J. A. Dionne, Revealing multiple classes of stable quantum emitters in hexagonal boron nitride with correlated optical and electron microscopy, Nat. Mater. 19, 534 (2020).

[20] S. Choi, T. T. Tran, C. Elbadawi, C. Lobo, X. Wang, S. Juodkazis, G. Seniutinas, M. Toth, and I. Aharonovich, Engineering and localization of quantum emitters in large hexagonal boron nitride layers, ACS Appl. Mater. Interfaces 8, 29642 (2016).

[21] Z. Xu, C. Elbadawi, T. T. Tran, M. Kianinia, X. Li, D. Liu, T. Hoffman, M. A. Nguyen, S. Kim, J. Edgar, X. Wu, L. Song, S. Ali, M. Ford, M. Toth, and igor aharonovich, Single photon emission from plasma treated 2D hexagonal boron nitride, Nanoscale 10, 7957 (2018).

[22] X. Li, G. D. Shepard, A. Cupo, N. Camporeale, K. Shayan, Y. Luo, V. Meunier, and S. Strauf, Nonmagnetic quantum emitters in boron nitride with ultranarrow and sideband-free emission spectra, ACS Nano 11, 6652 (2017).

[23] A. Gottscholl, M. Kianinia, V. Soltamov, S. Orlinskii, G. Mamin, C. Bradac, C. Kasper, K. Krambrock, A. Sperlich, M. Toth, I. Aharonovich, and V. Dyakonov, Initialization and read-out of intrinsic spin defects in a van Der waals crystal at room temperature, Nat. Mater. 19, 540 (2020).

[24] J. Ziegler, R. Klaiss, A. Blaikie, D. Miller, and V. R. Horowitz, Deterministic quantum emitter formation in hexagonal boron nitride via controlled edge creation, Nano Lett. 19, 2121 (2019).

[25] N. V. Proscia, Z. Shotan, H. Jayakumar, P. Reddy, C. Cohen, M. Dollar, A. Alkauskas, M. W. Doherty, C. A. Meriles, and V. M. Menon, Near-Deterministic activation of room-temperature quantum emitters in hexagonal boron nitride, Optica 5, 1128 (2018).

[26] T. T. Tran, D. Wang, Z. Q. Xu, A. Yang, M. Toth, T. W. Odom, and I. Aharonovich, Deterministic coupling of quantum emitters in 2D materials to plasmonic nanocavity arrays, Nano Lett. 17, 2634 (2017).

[27] T. T. Tran, M. Kianinia, M. Nguyen, S. Kim, Z. Q. Xu, A. Kubanek, M. Toth, and I. Aharonovich, Resonant excitation of quantum emitters in hexagonal boron nitride, ACS Photonics 5, 295 (2018).

[28] H. L. Stern, R. Wang, Y. Fan, R. Mizuta, J. C. Stewart, L. M. Needham, T. D. Roberts, R. Wai, N. S. Ginsberg, D. Klenerman, S. Hofmann, and S. F. Lee, Spectrally resolved photodynamics of individual emitters in large-area monolayers of hexagonal boron nitride, ACS Nano 13, 4538 (2019).

[29] Z. Shotan, H. Jayakumar, C. R. Considine, M. Mackoit, H. Fedder, J. Wrachtrup, A. Alkauskas, M. W. Doherty,
V. M. Menon, and C. A. Meriles, Photoinduced modification of single-photon emitters in hexagonal boron nitride, ACS Photonics 3, 2490 (2016).

[30] B. Spokoyny, H. Utzat, H. Moon, G. Grosso, D. Englund, and M. G. Bawendi, Effect of spectral diffusion on the coherence properties of a single quantum emitter in hexagonal boron nitride, J. Phys. Chem. Lett. 11, 1330 (2020).

[31] J. Wolters, N. Sadzak, A. W. Schell, T. Schroder, and O. Benson, Measurement of the Ultrafast Spectral Diffusion of the Optical Transition of Nitrogen Vacancy Centers in Nano-Size Diamond Using Correlation Interferometry, Phys. Rev. Lett. 110, 027401 (2013).

[32] A. Dietrich, M. Bürk, E. S. Steiger, L. Antoniuk, T. T. Tran, M. Nguyen, I. Aharonovich, F. Jelezko, and A. Kubanek, Observation of Fourier transform limited lines in hexagonal boron nitride, Phys. Rev. B 98, 081414(R) (2018).

[33] A. Dietrich, M. W. Doherty, I. Aharonovich, and A. Kubanek, Solid-State single photon source with Fourier transform limited lines at room temperature, Phys. Rev. B 101, 081401(R) (2020).

[34] B. Sontheimer, M. Braun, N. Nikolay, N. Sadzak, I. Aharonovich, and O. Benson, Photodynamics of quantum emitters in hexagonal boron nitride revealed by Lowtemperature spectroscopy, Phys. Rev. B 96, 121202(R) (2017).

[35] S. V. Novoselov, K. S. Geim, A. K. Morozov, S. V. Jiang, D. Zhang, Y. Dubonos, I. V. Grigorieva, and A. A. Firsov, Electric field effect in atomically thin carbon films, Science (80-.). 306, 666 (2004).

[36] X. F. Fan, W. T. Zheng, V. Chihaia, Z. X. Shen, and J. Kuo, Interaction between graphene and the surface of interaction between graphene and the surface of $\mathrm{SiO}_{2}$, J. Phys. Condens. Matter 24, 305004 (2012).

[37] P. Tonndorf, R. Schmidt, R. Schneider, J. Kern, M. Buscema, G. A. Steele, A. Castellanos-Gomez, H. S. J. van der Zant, S. Michaelis de Vasconcellos, and R. Bratschitsch, Single-Photon emission from localized excitons in an atomically thin semiconductor, Optica 2, 347 (2015).

[38] O. Iff, Y. M. He, N. Lundt, S. Stoll, V. Baumann, S. Höfling, and C. Schneider, Substrate engineering for high quality emission of free and localized excitons from atomic monolayers in hybrid architectures, Optica 4, 669 (2017).

[39] H. W. Cheng, C. T. Yuan, J. S. Wang, T. N. Lin, J. L. Shen, Y. J. Hung, J. Tang, and F. G. Tseng, Modification of photon emission statistics from single colloidal CdSe quantum dots by conductive materials, J. Phys. Chem. C 118, 18126 (2014).

[40] A. Sipahigil, K. D. Jahnke, L. J. Rogers, T. Teraji, J. Isoya, A. S. Zibrov, F. Jelezko, and M. D. Lukin, Indistinguishable Photons From Separated Silicon-Vacancy Centers in Diamond, Phys. Rev. Lett. 113, 113602 (2014).

[41] See Supplemental Material at http://link.aps.org/sup plemental/10.1103/PhysRevApplied.15.014036 for more details about analysis, methods, and materials.

[42] L. Novotny and B. Hecht, in Principles of Nano-Optics (Cambridge University Press, Cambridge, England, 2006), pp. 282-312.

[43] A. A. Maradudin, Theoretical and experimental aspects of the effects of point defects and disorder on the vibrations of crystals, Solid State Phys. 18, 273 (1966). 
[44] V. Hizhnyakov, H. Kaasik, and I. Sildos, Zero-Phonon lines: The effect of a strong softening of elastic springs in the excited state, Phys. Status Solidi Basic Res. 234, 644 (2002).

[45] N. R. Jungwirth, B. Calderon, Y. Ji, M. G. Spencer, M. E. Flatté, and G. D. Fuchs, Temperature dependence of wavelength selectable zero-phonon emission from single defects in hexagonal boron nitride, Nano Lett. 16, 6052 (2016).

[46] D. Wigger, R. Schmidt, O. Del Pozo-Zamudio, J. A. Preuß, P. Tonndorf, R. Schneider, P. Steeger, J. Kern, Y. Khodaei, J. Sperling, S. M. De Vasconcellos, R. Bratschitsch, and T. Kuhn, Phonon-Assisted emission and absorption of individual color centers in hexagonal boron nitride, 2D Mater. 6, 035006 (2019).

[47] F. Cadiz, E. Courtade, C. Robert, G. Wang, Y. Shen, H. Cai, T. Taniguchi, K. Watanabe, H. Carrere, D. Lagarde, M. Manca, T. Amand, P. Renucci, S. Tongay, X. Marie, and B. Urbaszek, Excitonic Linewidth Approaching the
Homogeneous Limit in $\mathrm{MoS}_{2}$-Based van Der Waals Heterostructures, Phys. Rev. X. 7, 021026 (2017).

[48] P. Dey, J. Paul, Z. Wang, C. E. Stevens, C. Liu, A. H. Romero, J. Shan, D. J. Hilton, and D. Karaiskaj, Optical Coherence in Atomic-Monolayer Transition-Metal Dichalcogenides Limited by Electron-Phonon Interactions, Phys. Rev. Lett. 116, 127402 (2016).

[49] L. Vinna, S. I. Logothetidis, and M. Cardona, Temperature dependence of the dielectric function of germanium, Phys. Rev. B 30, 1979 (1984).

[50] H. Y. Chen, D. Sangalli, and M. Bernardi, Exciton-Phonon Interaction and Relaxation Times From First Principles, Phys. Rev. Lett. 125, 107401 (2020).

[51] W. H. Lin, V. W. Brar, D. Jariwala, M. C. Sherrott, W. S. Tseng, C. I. Wu, N. C. Yeh, and H. A. Atwater, AtomicScale structural and chemical characterization of hexagonal boron nitride layers synthesized at the wafer-scale with monolayer thickness control, Chem. Mater. 29, 4700 (2017). 